\title{
Carbon-Hydrogen Bond Activation: Two, Three or More Mechanisms?
}

Benjamin Alan Vastine and Michael B. Hall*

Department of Chemistry, Texas A\&M University, P.O. Box 30012, College Station, TX, USA, 77841-3255

\section{Supporting Information}

Optimized geometry, AIM data, and coordinates of $\mathbf{1}$.

73.5

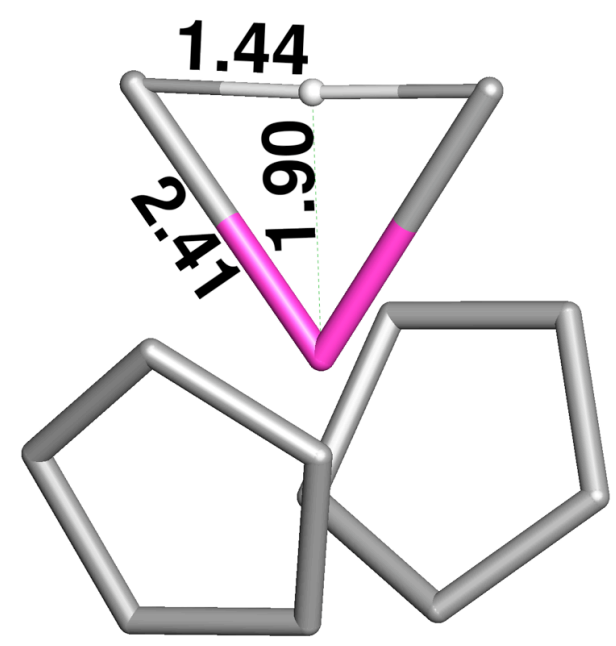

$\mathrm{E}_{S C F}=-1228.30604309$ hartrees

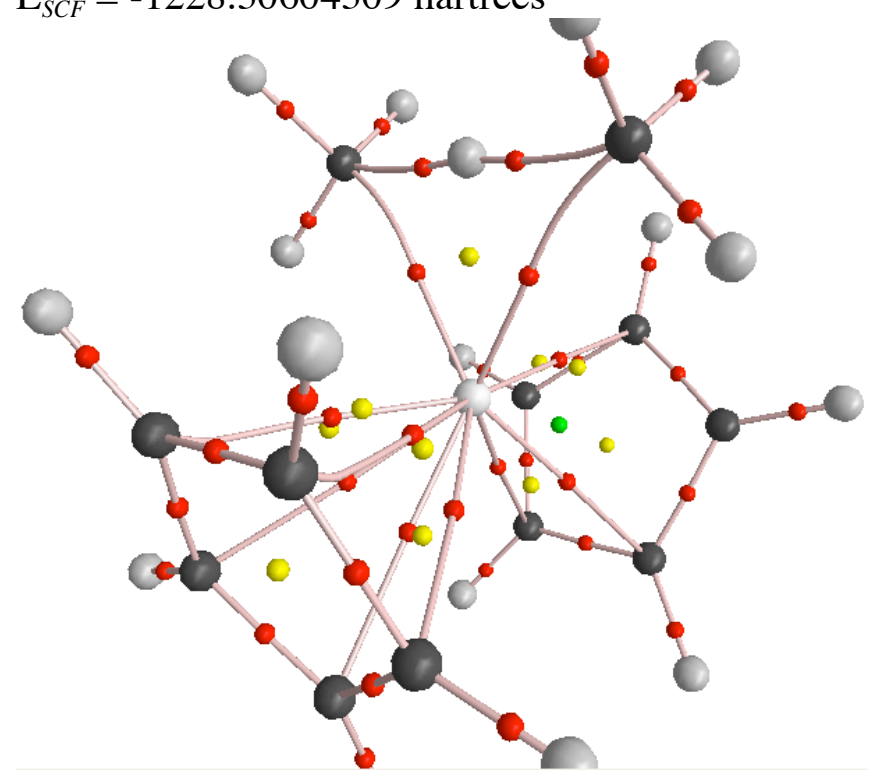




$\begin{array}{lccc}21 & -0.005496 & 0.186358 & -0.026992 \\ 6 & 0.035628 & 1.895314 & -1.722630 \\ 6 & -0.015396 & 2.299429 & 1.127708 \\ 1 & 0.002570 & 2.065088 & -0.293039 \\ 6 & -1.908742 & -0.844553 & 1.240910 \\ 6 & -2.431533 & 0.334754 & 0.638351 \\ 6 & -2.414493 & 0.153491 & -0.767404 \\ 6 & 2.515718 & 0.478025 & -0.010971 \\ 6 & 2.215203 & -0.436226 & -1.054853 \\ 6 & 1.672064 & -1.610718 & -0.469466 \\ 6 & -1.583903 & -1.758828 & 0.203840 \\ 6 & -1.881803 & -1.138719 & -1.038352 \\ 6 & 2.166203 & -0.133078 & 1.222244 \\ 6 & 1.641925 & -1.422778 & 0.942116 \\ 1 & -1.793901 & -1.021813 & 2.306848 \\ 1 & -2.776705 & 1.219102 & 1.163320 \\ 1 & -2.744173 & 0.874686 & -1.507662 \\ 1 & 2.924042 & 1.475853 & -0.135111 \\ 1 & 2.365175 & -0.261963 & -2.116269 \\ 1 & 1.345936 & -2.498207 & -1.003003 \\ 1 & 0.966933 & 2.422146 & -1.969876 \\ 1 & -0.021391 & 1.019302 & -2.395161 \\ 1 & -0.812146 & 2.541705 & -1.986934 \\ 1 & -1.165595 & -2.751276 & 0.336772 \\ 1 & -1.742154 & -1.580207 & -2.021456 \\ 1 & 2.272344 & 0.313676 & 2.206460 \\ 1 & 1.288462 & -2.141546 & 1.674951 \\ 1 & -0.095320 & 1.644555 & 2.015186 \\ 1 & 0.907345 & 2.882379 & 1.249045 \\ 1 & -0.871230 & 2.986333 & 1.173805\end{array}$

Basis Sets

Opt and Freq

Sc: LANL2DZ(f)

C: aug-cc-pvdz

$\mathrm{H}$ being transferred: aug-cc-pvdz

All other H: D95.

AIM analysis

Sc: WTBS

C: aug-cc-pvdz

$\mathrm{H}$ being transferred: aug-cc-pvdz

All other H: D95. 


\begin{tabular}{|c|c|c|c|}
\hline & $\rho$ (a.u.) & $\nabla^{2}(\rho)$ & Distance $(\AA)$ \\
\hline Sc-C & 0.049837 & 0.151443 & 1.157927 \\
\hline $\mathrm{C}-\mathrm{H}_{\mathrm{t}}$ & 0.128339 & -0.156703 & 0.966571 \\
\hline $\mathrm{C}-\mathrm{H}$ & 0.259873 & -0.680968 & 0.685212 \\
\hline Ring & 0.046071 & 0.209364 & 1.136153 \\
\hline
\end{tabular}

Optimized geometry, AIM data, and coordinates of $\mathbf{2}$.

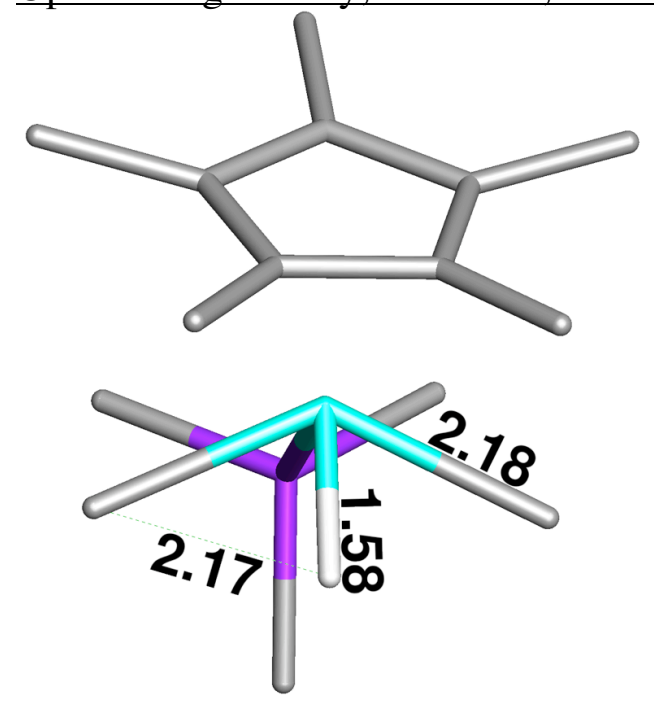

130.3

$\mathrm{E}_{S C F}=-1036.17995683$ hartrees
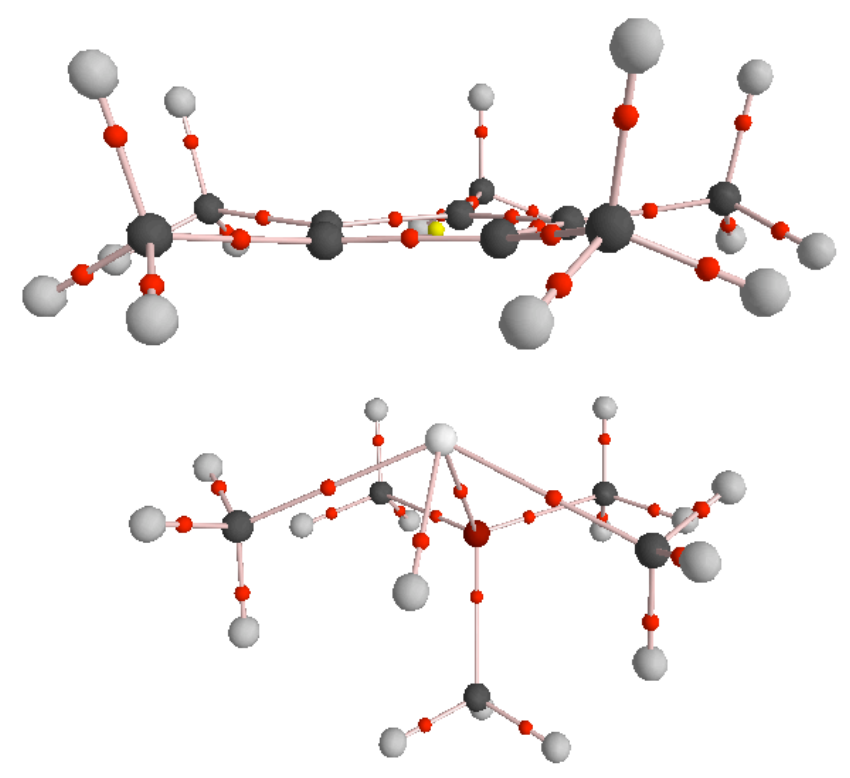


$\begin{array}{lrrr}77 & -0.005728 & -0.008154 & -0.477058 \\ 15 & 2.299309 & -0.000346 & 0.046256 \\ 6 & 0.489846 & -1.996015 & -1.222500 \\ 6 & 0.509259 & 1.960681 & -1.259607 \\ 1 & 0.115587 & -0.016721 & -2.051335 \\ 6 & -1.413237 & 1.182475 & 0.955599 \\ 6 & -0.978033 & 0.048624 & 1.718277 \\ 6 & -1.389030 & -1.136578 & 1.023657 \\ 6 & -2.126351 & -0.735556 & -0.167329 \\ 6 & -2.140114 & 0.698722 & -0.209876 \\ 6 & -1.320729 & 2.617351 & 1.391861 \\ 6 & -0.321590 & 0.096757 & 3.071083 \\ 6 & -1.267867 & -2.541661 & 1.542966 \\ 6 & -2.905797 & -1.651269 & -1.070499 \\ 6 & -2.934560 & 1.545244 & -1.166283 \\ 6 & 2.846784 & -1.398875 & 1.135825 \\ 6 & 3.459651 & -0.088692 & -1.392286 \\ 6 & 2.892520 & 1.489404 & 0.981757 \\ 1 & -0.337099 & -2.350229 & -1.851989 \\ 1 & 1.391131 & -2.007521 & -1.839011 \\ 1 & 0.624845 & -2.678589 & -0.378831 \\ 1 & -0.351562 & -2.693278 & 2.123899 \\ 1 & -2.114343 & -2.769843 & 2.207456 \\ 1 & -1.284217 & -3.284091 & 0.739516 \\ 1 & 0.289402 & -0.790227 & 3.268547 \\ 1 & 0.308682 & 0.983516 & 3.197975 \\ 1 & -1.086571 & 0.136568 & 3.860615 \\ 1 & -0.417719 & 2.816909 & 1.979126 \\ 1 & -1.333622 & 3.310924 & 0.545812 \\ 1 & -2.182056 & 2.870416 & 2.027738 \\ 1 & -3.040202 & 1.067211 & -2.145238 \\ 1 & -3.947160 & 1.711412 & -0.770439 \\ 1 & -2.486399 & 2.531634 & -1.321657 \\ 1 & -2.448848 & -2.642060 & -1.154466 \\ 1 & -3.920559 & -1.797596 & -0.672376 \\ 1 & -3.006561 & -1.241730 & -2.080615 \\ 1 & -0.336684 & 2.325690 & -1.857950 \\ 1 & 0.693552 & 2.652292 & -0.422846 \\ 1 & 1.387320 & 1.946981 & -1.910219 \\ 1 & 2.939997 & 1.345543 & 1.294252 \\ 1 & 2.274914 & 2.391719 & 0.355156 \\ 1 & & 1.638082 & 1.875769 \\ 1 & 0.709449 & -2.109859 \\ 1 & -36599 & 0.023079 & -1.045279 \\ 1 & -1.052317 & -1.904975 \\ 1 & -1.354047 & 2.092283\end{array}$




$\begin{array}{llll}1 & 2.639630 & -2.367543 & 0.658963 \\ 1 & 3.928309 & -1.326256 & 1.333738\end{array}$

Basis Sets

Opt and Freq

Ir: LANL2DZ(f)

P: cc-pvdz

Aromatic carbons of $\mathrm{Cp} *$ ring: cc-pvdz

Carbon atoms of methyl ligands: cc-pvdz

$\mathrm{H}$ being transferred: cc-pvdz

All other C and H: D95.

AIM analysis

Ir: WTBS

P: cc-pvdz

Aromatic carbons of $\mathrm{Cp} *$ ring: cc-pvdz

Carbon atoms of methyl ligands: cc-pvdz

$\mathrm{H}$ being transferred: cc-pvdz

All other C and H: D95.

AIM CP data

\begin{tabular}{|c|c|c|c|}
\hline & $\rho$ (a.u.) & $\nabla^{2}$ & Distance $(\AA)$ \\
\hline Ir-C & 0.104164 & 0.093511 & 1.192596 \\
\hline Ir-H & 0.162408 & 0.019232 & 1.093013 \\
\hline
\end{tabular}

Optimized geometry, AIM data, and coordinates of $\mathbf{3}$.

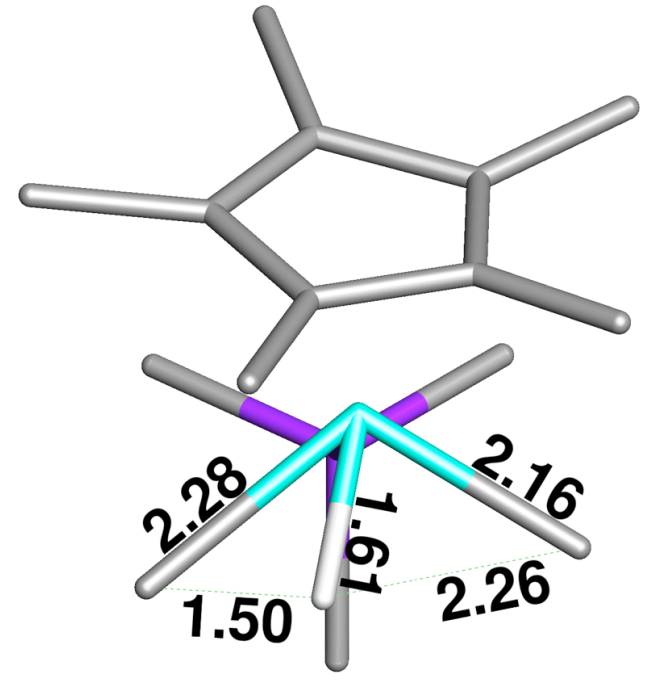

$$
110.0
$$

$\mathrm{E}_{S C F}=-1036.16860046$ hartrees 


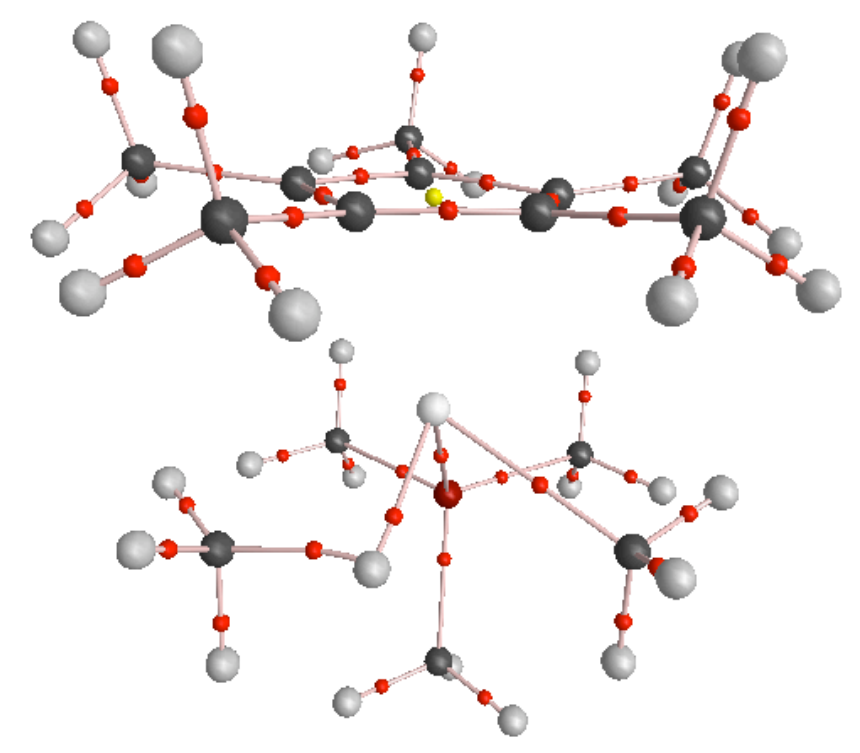

$\begin{array}{lrrr}77 & 0.022537 & -0.022930 & -0.442191 \\ 15 & -2.269808 & -0.023198 & 0.070644 \\ 6 & -0.476372 & 1.680434 & -1.665509 \\ 6 & -0.406365 & -1.951050 & -1.579924 \\ 1 & -0.123072 & -0.531550 & -1.960322 \\ 6 & 1.543369 & -1.133280 & 0.950578 \\ 6 & 0.909301 & -0.122604 & 1.729569 \\ 6 & 1.146858 & 1.161177 & 1.090291 \\ 6 & 2.033353 & 0.930763 & -0.041272 \\ 6 & 2.233490 & -0.480912 & -0.158541 \\ 6 & 1.607057 & -2.602821 & 1.263634 \\ 6 & 0.222977 & -0.317717 & 3.053634 \\ 6 & 0.819325 & 2.511359 & 1.671755 \\ 6 & 2.705514 & 1.998509 & -0.857187 \\ 6 & 3.137782 & -1.175462 & -1.140357 \\ 6 & -2.860385 & 1.503886 & 0.930880 \\ 6 & -3.451625 & -0.188911 & -1.347142 \\ 6 & -2.813685 & -1.393991 & 1.192620 \\ 1 & 0.354387 & 1.877360 & -2.350253 \\ 1 & -1.378294 & 1.562596 & -2.273395 \\ 1 & -0.609056 & 2.554684 & -1.017394 \\ 1 & -0.045017 & 2.472719 & 2.342750 \\ 1 & 1.667135 & 2.895093 & 2.257880 \\ 1 & 0.605825 & 3.252824 & 0.893858 \\ 1 & -0.617073 & 0.370318 & 3.199555 \\ 1 & -0.142401 & -1.340696 & 3.186931 \\ 1 & 0.934122 & -0.123176 & 3.869990 \\ 1 & 0.785831 & -2.922680 & 1.913474 \\ 1 & 1.577238 & -3.220010 & 0.358389 \\ 1 & 2.545248 & -2.846825 & 1.782961\end{array}$




$\begin{array}{lrrr}1 & 3.247494 & -0.608768 & -2.070283 \\ 1 & 4.142751 & -1.298747 & -0.711093 \\ 1 & 2.777557 & -2.177905 & -1.397117 \\ 1 & 2.069159 & 2.878622 & -0.992972 \\ 1 & 3.621371 & 2.334710 & -0.349289 \\ 1 & 2.996883 & 1.638315 & -1.849006 \\ 1 & 0.491147 & -2.340986 & -2.067623 \\ 1 & -0.646950 & -2.585976 & -0.723078 \\ 1 & -1.237938 & -1.992558 & -2.288949 \\ 1 & -3.894447 & -1.335628 & 1.370626 \\ 1 & -2.587008 & -2.364477 & 0.736679 \\ 1 & -2.296172 & -1.329817 & 2.154255 \\ 1 & -3.326061 & -1.157855 & -1.841722 \\ 1 & -4.481984 & -0.119773 & -0.977910 \\ 1 & -3.289971 & 0.603465 & -2.083781 \\ 1 & -2.311115 & 1.644705 & 1.867816 \\ 1 & -2.690080 & 2.378704 & 0.294540 \\ 1 & -3.931284 & 1.430639 & 1.155362\end{array}$

$\underline{\text { Basis Sets }}$

Opt and Freq

Ir: LANL2DZ(f)

P: cc-pvdz

Aromatic carbons of $\mathrm{Cp}^{*}$ ring: cc-pvdz

Carbon atoms of methyl ligands: cc-pvdz

$\mathrm{H}$ being transferred: cc-pvdz

All other C and H: D95.

AIM analysis

Ir: WTBS

P: cc-pvdz

Aromatic carbons of $\mathrm{Cp}$ * ring: cc-pvdz

Carbon atoms of methyl ligands: cc-pvdz

$\mathrm{H}$ being transferred: cc-pvdz

All other C and H: D95.

AIM data

\begin{tabular}{|c|c|c|c|}
\hline & $\rho$ (a.u.) & $\nabla^{2}$ & Distance $(\AA)$ \\
\hline Ir-C' & 0.108916 & 0.111959 & 1.175042 \\
\hline Ir-H & 0.162408 & 0.019232 & 1.093013 \\
\hline C-H & 0.117912 & -0.069848 & 0.922356 \\
\hline
\end{tabular}


Optimized geometry, AIM data, and coordinates of $\mathbf{4}$

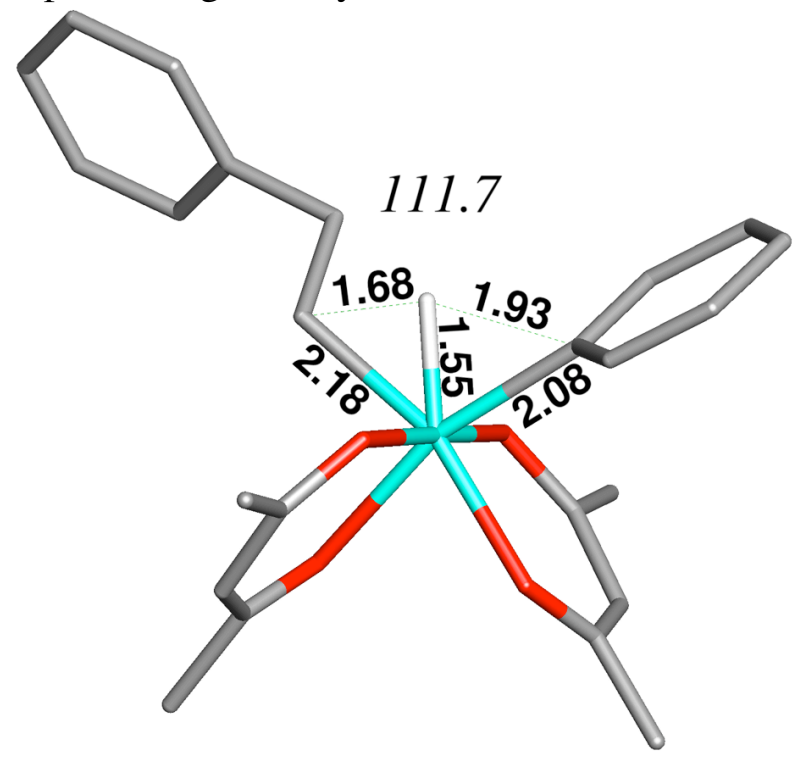

$\mathrm{E}_{S C F}=-1337.54215904$ hartrees

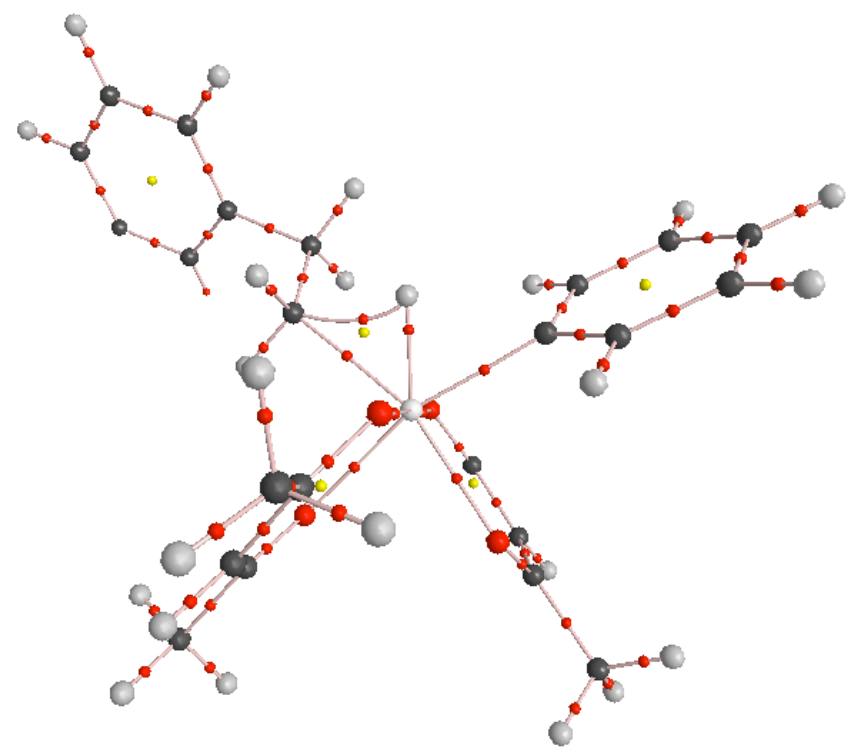

Opt and Freq

Ir: LANL2DZ(f)

O: cc-pvdz

Ligated carbons: cc-pvdz

$\mathrm{H}$ being transferred: cc-pvdz

All other C and H: D95.

AIM analysis

Ir: WTBS 
O: cc-pvdz

Ligated carbons: cc-pvdz

$\mathrm{H}$ being transferred: cc-pvdz

All other C and H: D95.

$\begin{array}{lccc}77 & 0.492224 & 0.128681 & -0.112894 \\ 8 & 0.786896 & 1.072737 & -1.906841 \\ 8 & -0.047066 & 1.964242 & 0.865324 \\ 8 & 2.406325 & 0.816717 & 0.423952 \\ 8 & 0.172802 & -0.759010 & 1.699934 \\ 6 & -1.654602 & 0.016879 & -0.489858 \\ 6 & 1.473216 & -1.569195 & -0.796941 \\ 1 & -0.341856 & -0.917614 & -0.903251 \\ 6 & 0.673745 & 2.351390 & -2.067998 \\ 6 & 0.309315 & 3.316405 & -1.102393 \\ 6 & -0.014251 & 3.097351 & 0.261339 \\ 6 & 2.933541 & 0.555903 & 1.567925 \\ 6 & 2.328319 & -0.154996 & 2.634221 \\ 6 & 1.041064 & -0.733513 & 2.659705 \\ 6 & 0.994514 & 2.806512 & -3.481530 \\ 6 & -0.370909 & 4.291740 & 1.131433 \\ 6 & 4.348188 & 1.079793 & 1.741627 \\ 6 & 0.561153 & -1.421778 & 3.925076 \\ 6 & -3.942667 & -0.965069 & -0.015285 \\ 6 & -4.767912 & -1.380199 & -1.083683 \\ 6 & -6.150142 & -1.108859 & -1.079180 \\ 6 & -6.730954 & -0.414933 & -0.000416 \\ 6 & -5.918553 & 0.003251 & 1.072837 \\ 6 & -4.538701 & -0.270338 & 1.062962 \\ 6 & -2.442993 & -1.231179 & -0.030406 \\ 6 & 1.235121 & -2.846019 & -0.251884 \\ 6 & 2.468563 & -1.440253 & -1.783696 \\ 6 & 3.227522 & -2.554101 & -2.196933 \\ 6 & 2.994050 & -3.824472 & -1.635773 \\ 6 & 1.989507 & -3.964921 & -0.659486 \\ 1 & 0.345525 & 2.281033 & -4.193117 \\ 1 & 0.866756 & 3.884978 & -3.606208 \\ 1 & 2.029808 & 2.537303 & -3.727118 \\ 1 & -1.382878 & 4.160850 & 1.535355 \\ 1 & 0.315240 & 4.334996 & 1.986907 \\ 1 & -0.322345 & 5.236974 & 0.583876 \\ 1 & 5.021937 & 0.518936 & 1.080158 \\ 1 & 4.392080 & 2.131592 & 1.435074 \\ 1 & 4.703059 & 0.983537 & 2.771407 \\ 1 & 0.287244 & -2.460036 & 3.699334 \\ 1 & 1.321613 & -1.414649 & 4.710260\end{array}$




$\begin{array}{lrrr}1 & -0.341350 & -0.919621 & 4.296588 \\ 1 & 0.278885 & 4.344070 & -1.444924 \\ 1 & 2.919012 & -0.263439 & 3.536251 \\ 1 & -4.327769 & -1.921129 & -1.920024 \\ 1 & -6.769579 & -1.439954 & -1.909890 \\ 1 & -7.798404 & -0.206439 & 0.006391 \\ 1 & -6.359738 & 0.534876 & 1.913241 \\ 1 & -3.918680 & 0.051273 & 1.898517 \\ 1 & -1.948788 & 0.877906 & 0.118326 \\ 1 & -1.861703 & 0.250585 & -1.541483 \\ 1 & -2.228165 & -2.079121 & -0.696685 \\ 1 & -2.096061 & -1.512282 & 0.971667 \\ 1 & 0.464003 & -2.977733 & 0.503500 \\ 1 & 1.787175 & -4.938658 & -0.216962 \\ 1 & 3.578013 & -4.685087 & -1.954937 \\ 1 & 3.996949 & -2.424848 & -2.956217 \\ 1 & 2.660305 & -0.470606 & -2.232133\end{array}$

AIM Data

\begin{tabular}{|c|c|c|c|}
\hline & $\rho$ (a.u.) & $\nabla^{2}$ & Distance $(\AA)$ \\
\hline $\mathrm{Ir}-\mathrm{C}_{\mathrm{sp}} 2$ & 0.107763 & 0.131764 & 1.228019 \\
\hline $\mathrm{Ir}-\mathrm{C}_{\mathrm{sp}} 3$ & 0.089130 & 0.127722 & 1.241145 \\
\hline $\mathrm{Ir}_{-} \mathrm{H}_{\mathrm{t}}$ & 0.167010 & 0.013086 & 1.231745 \\
\hline $\mathrm{C}_{\mathrm{sp}} 3-\mathrm{H}_{\mathrm{t}}$ & 0.094083 & 0.033759 & 1.043281 \\
\hline $\mathrm{Ring}$ & 0.092980 & 0.128984 & 1.350846 \\
\hline
\end{tabular}

Optimized geometry, AIM data, and coordinates of $\mathbf{5}$ C2 symmetric

$$
116.9
$$

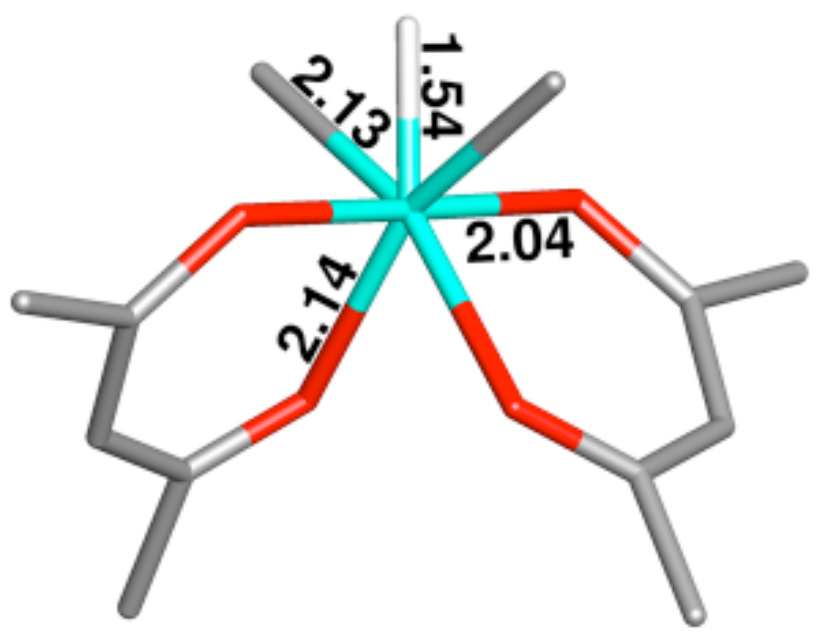


$\mathrm{E}_{S C F}=-875.509732042$ hartrees

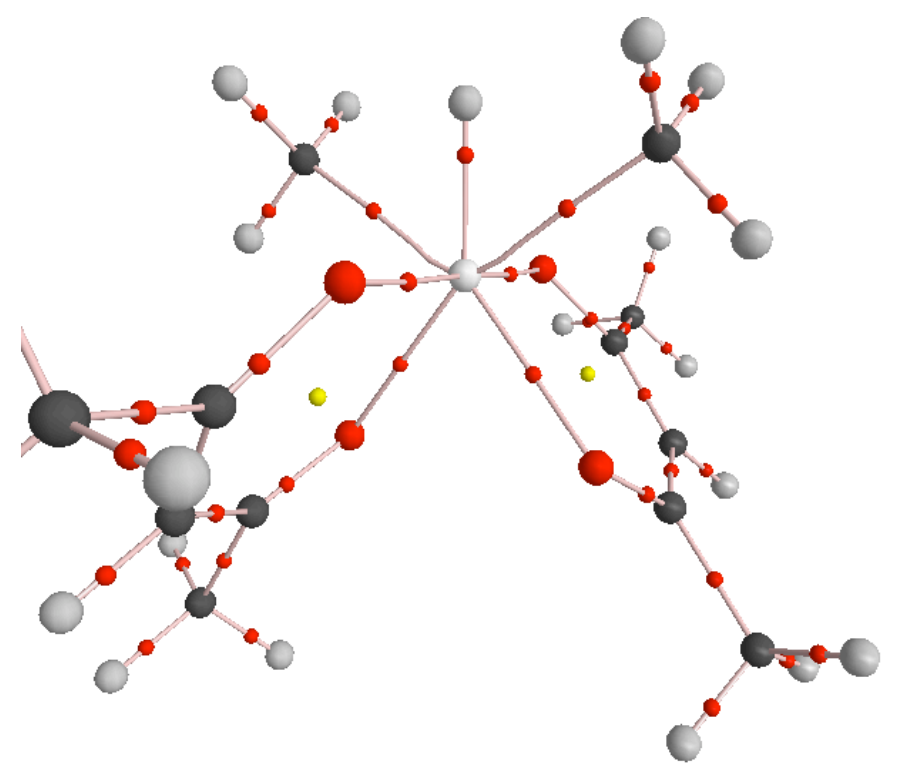

$\underline{\text { Basis Sets }}$

Opt and Freq

Ir: LANL2DZ(f)

O: cc-pvdz

Ligated carbons: cc-pvdz

$\mathrm{H}$ being transferred: $c c-p v d z$

All other C and H: D95.

AIM analysis

Ir: WTBS

O: cc-pvdz

Ligated carbons: cc-pvdz

$\mathrm{H}$ being transferred: cc-pvdz

All other C and H: D95.

AIM Data

\begin{tabular}{|c|c|c|c|}
\hline & $\rho$ (a.u.) & $\nabla^{2}$ & Distance $(\AA)$ \\
\hline Ir-C & 0.115063 & 0.097341 & 1.162684 \\
\hline Ir-H & 0.174558 & -0.026269 & 1.084151 \\
\hline
\end{tabular}

$\begin{array}{lrrc}77 & 0.000000 & 0.000000 & 0.710896 \\ 8 & -0.214629 & 2.029753 & 0.721039 \\ 8 & 1.350389 & 0.035809 & -0.945508 \\ 8 & -1.350389 & -0.035809 & -0.945508 \\ 8 & 0.214629 & -2.029753 & 0.721039\end{array}$




$\begin{array}{lrcc}6 & 1.808213 & 0.121364 & 1.824017 \\ 6 & -1.808213 & -0.121364 & 1.824017 \\ 6 & 0.355786 & 2.806787 & -0.138095 \\ 6 & 1.234944 & 2.438410 & -1.183433 \\ 6 & 1.669347 & 1.134849 & -1.531847 \\ 6 & -1.669347 & -1.134849 & -1.531847 \\ 6 & -1.234944 & -2.438410 & -1.183433 \\ 6 & -0.355786 & -2.806787 & -0.138095 \\ 6 & 0.000000 & 4.272342 & 0.045668 \\ 6 & 2.617727 & 0.967567 & -2.708409 \\ 6 & -2.617727 & -0.967567 & -2.708409 \\ 6 & 0.000000 & -4.272342 & 0.045668 \\ 1 & 0.216495 & 4.578166 & 1.076643 \\ 1 & 0.546876 & 4.919187 & -0.645532 \\ 1 & -1.077434 & 4.408173 & -0.114290 \\ 1 & 3.548131 & 0.498476 & -2.363418 \\ 1 & 2.168067 & 0.288759 & -3.444092 \\ 1 & 2.853606 & 1.918898 & -3.193244 \\ 1 & -3.548131 & -0.498476 & -2.363418 \\ 1 & -2.168067 & -0.288759 & -3.444092 \\ 1 & -2.853606 & -1.918898 & -3.193244 \\ 1 & -0.216495 & -4.578166 & 1.076643 \\ 1 & -0.546876 & -4.919187 & -0.645532 \\ 1 & 1.077434 & -4.408173 & -0.114290 \\ 1 & 1.613014 & 3.249448 & -1.794792 \\ 1 & -1.613014 & -3.249448 & -1.794792 \\ 1 & -1.876749 & -1.030175 & 2.432286 \\ 1 & -1.964166 & 0.762594 & 2.451786 \\ 1 & -2.578173 & -0.138350 & 1.046094 \\ 1 & 2.578173 & 0.138350 & 1.046094 \\ 1 & 1.876749 & 1.030175 & 2.432286 \\ 1 & 1.964166 & -0.762594 & 2.451786 \\ 1 & 0.000000 & 0.000000 & 2.254810\end{array}$


Optimized geometry, AIM data, and coordinates of $\mathbf{6}$

C2 symmetric

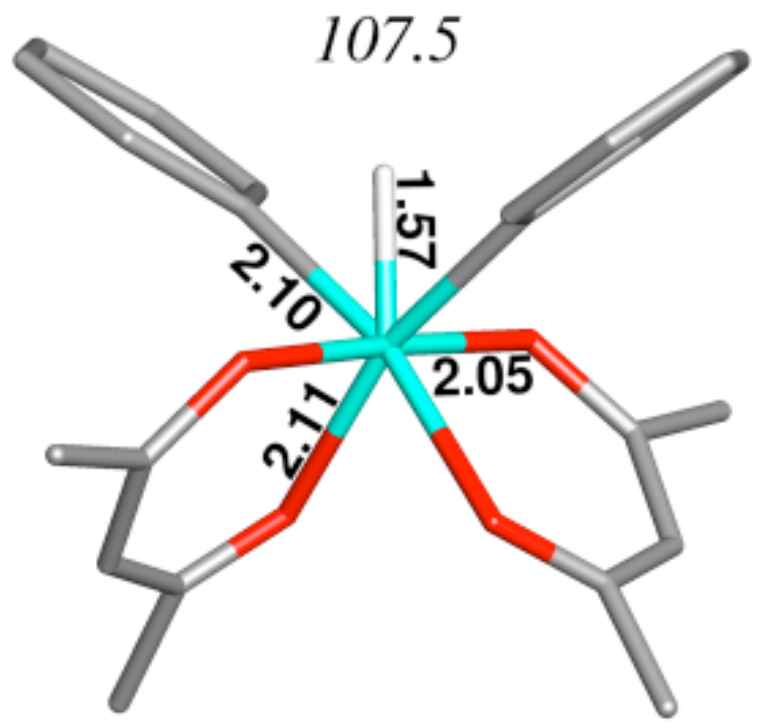

$\mathrm{E}_{S C F}=-1259.01122033$ hartrees

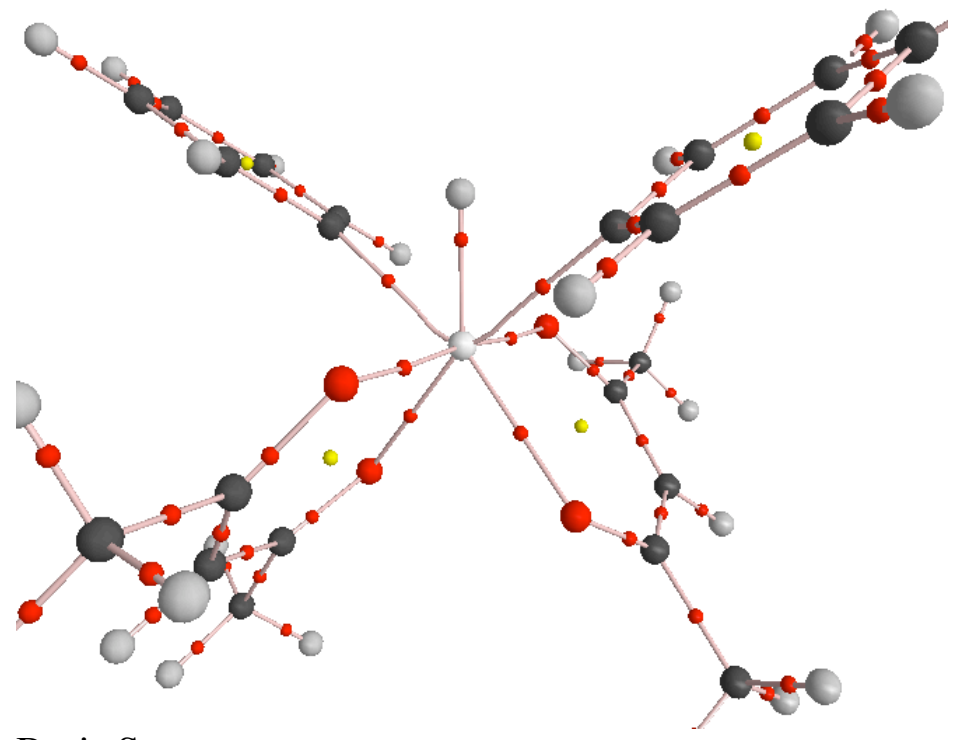

$\underline{\text { Basis Sets }}$

Opt and Freq

Ir: LANL2DZ(f)

O: cc-pvdz

phenyl carbons: cc-pvdz

$\mathrm{H}$ being transferred: cc-pvdz

All other C and H: D95.

AIM analysis

Ir: WTBS 
O: cc-pvdz

phenyl carbons: cc-pvdz

$\mathrm{H}$ being transferred: cc-pvdz

All other C and H: D95.

AIM Data
\begin{tabular}{|c|c|c|c|}
\hline & $\rho$ (a.u.) & $\nabla^{2}$ & Distance $(\AA)$ \\
\hline Ir-C & 0.120961 & 0.143555 & 1.136592 \\
\hline Ir-H $\mathrm{H}_{\mathrm{t}}$ & 0.159288 & 0.019252 & 1.104014 \\
\hline
\end{tabular}

$\begin{array}{lrrr}77 & 0.000000 & 0.000000 & 0.010029 \\ 8 & 0.000000 & 2.047634 & -0.039124 \\ 8 & 1.374312 & -0.143426 & -1.588328 \\ 8 & -1.374312 & 0.143426 & -1.588328 \\ 8 & 0.000000 & -2.047634 & -0.039124 \\ 6 & 1.695168 & 0.008689 & 1.252015 \\ 6 & -1.695168 & -0.008689 & 1.252015 \\ 6 & 0.659080 & 2.732597 & -0.919144 \\ 6 & 1.526174 & 2.242429 & -1.918035 \\ 6 & 1.842902 & 0.887856 & -2.194156 \\ 6 & -1.842902 & -0.887856 & -2.194156 \\ 6 & -1.526174 & -2.242429 & -1.918035 \\ 6 & -0.659080 & -2.732597 & -0.919144 \\ 6 & 0.440263 & 4.230411 & -0.797463 \\ 6 & 2.832056 & 0.572334 & -3.302427 \\ 6 & -2.832056 & -0.572334 & -3.302427 \\ 6 & -0.440263 & -4.230411 & -0.797463 \\ 6 & 2.503640 & -1.140533 & 1.318139 \\ 6 & 3.716825 & -1.117773 & 2.015305 \\ 6 & 4.137643 & 0.042993 & 2.674107 \\ 6 & 3.328591 & 1.182388 & 2.635448 \\ 6 & 2.115257 & 1.164033 & 1.936475 \\ 6 & -2.503640 & 1.140533 & 1.318139 \\ 6 & -3.716825 & 1.117773 & 2.015305 \\ 6 & -4.137643 & -0.042993 & 2.674107 \\ 6 & -3.328591 & -1.182388 & 2.635448 \\ 6 & -2.115257 & -1.164033 & 1.936475 \\ 1 & 0.000000 & 0.000000 & 1.582518 \\ 1 & 0.751606 & 4.569649 & 0.198872 \\ 1 & 0.995632 & 4.789942 & -1.554665 \\ 1 & -0.629265 & 4.454250 & -0.897893 \\ 1 & 3.719855 & 0.095103 & -2.867514 \\ 1 & 2.385110 & -0.147513 & -3.999053 \\ 1 & 3.141419 & 1.465810 & -3.851661 \\ 1 & -3.719855 & -0.095103 & -2.867514 \\ 6 & & & \\ 6 & & & \end{array}$




$\begin{array}{lrrr}1 & -2.385110 & 0.147513 & -3.999053 \\ 1 & -3.141419 & -1.465810 & -3.851661 \\ 1 & -0.751606 & -4.569649 & 0.198872 \\ 1 & -0.995632 & -4.789942 & -1.554665 \\ 1 & 0.629265 & -4.454250 & -0.897893 \\ 1 & 2.004785 & 2.986521 & -2.543662 \\ 1 & -2.004785 & -2.986521 & -2.543662 \\ 1 & 1.489598 & 2.053576 & 1.925888 \\ 1 & 3.636413 & 2.089752 & 3.153594 \\ 1 & 5.080368 & 0.055568 & 3.218423 \\ 1 & 4.335920 & -2.013818 & 2.042988 \\ 1 & 2.184689 & -2.048604 & 0.815328 \\ 1 & -1.489598 & -2.053576 & 1.925888 \\ 1 & -3.636413 & -2.089752 & 3.153594 \\ 1 & -5.080368 & -0.055568 & 3.218423 \\ 1 & -4.335920 & 2.013818 & 2.042988 \\ 1 & -2.184689 & 2.048604 & 0.815328\end{array}$

Optimized geometry, AIM data, and coordinates of $\mathbf{7}$ C2 symmetric

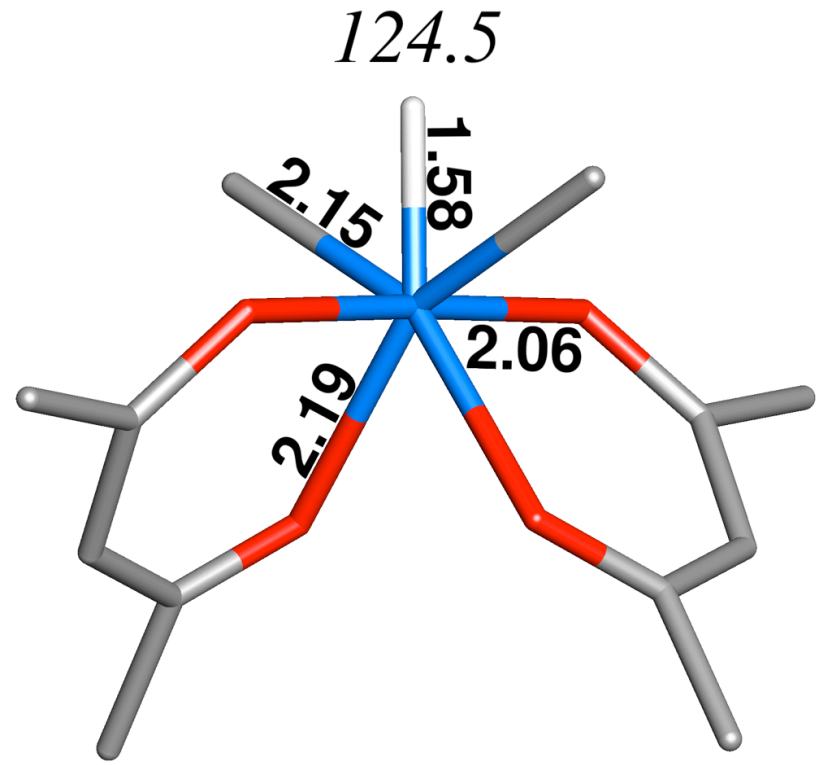

$\mathrm{E}_{S C F}=-861.903595382$ hartrees 


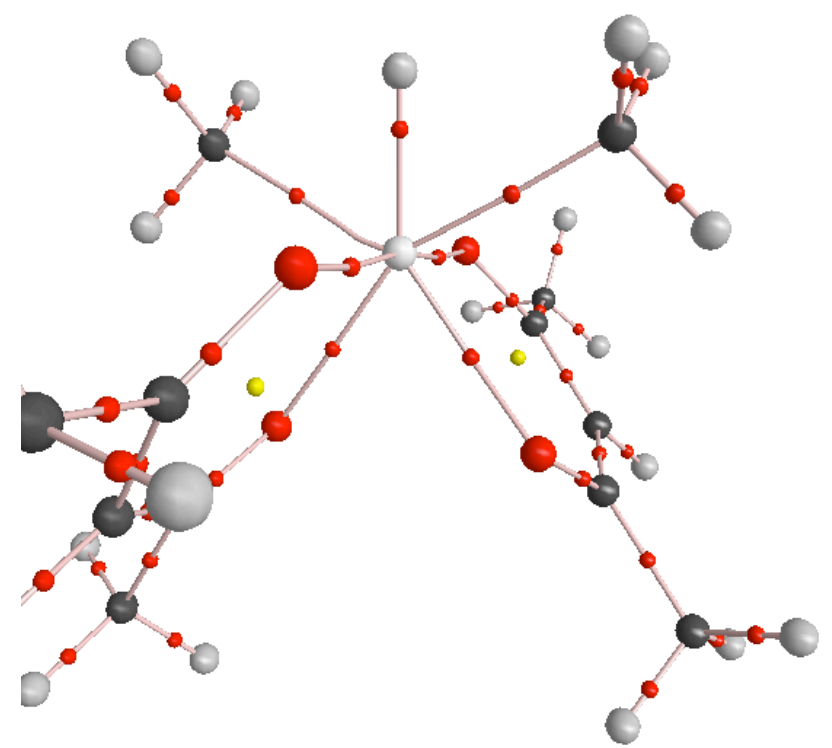

$\underline{\text { Basis Sets }}$

Opt and Freq

Os: LANL2DZ(f)

O: cc-pvdz

Ligated carbons: cc-pvdz

$\mathrm{H}$ being transferred: cc-pvdz

All other C and H: D95.

AIM analysis

Os: WTBS

O: cc-pvdz

Ligated carbons: cc-pvdz

$\mathrm{H}$ being transferred: cc-pvdz

All other C and H: D95.

AIM Data

\begin{tabular}{|c|c|c|c|}
\hline & $\rho$ (a.u.) & $\nabla^{2}$ & Distance $(\AA)$ \\
\hline Os-C & 0.112665 & 0.1309667 & 1.145907 \\
\hline Os- $_{\mathrm{t}}$ & 0.161597 & 0.0465918 & 1.086563 \\
\hline
\end{tabular}

$\begin{array}{lrrr}76 & 0.000000 & 0.000000 & 0.758846 \\ 8 & -0.193255 & 2.049114 & 0.719066 \\ 8 & 1.339828 & 0.039566 & -0.967319 \\ 8 & -1.339828 & -0.039566 & -0.967319 \\ 8 & 0.193255 & -2.049114 & 0.719066 \\ 6 & 1.892800 & 0.185030 & 1.759041 \\ 6 & -1.892800 & -0.185030 & 1.759041 \\ 6 & 0.362018 & 2.823638 & -0.137140 \\ 6 & 1.241391 & 2.448599 & -1.194338\end{array}$




$\begin{array}{lrrr}6 & 1.655342 & 1.140865 & -1.545857 \\ 6 & -1.655342 & -1.140865 & -1.545857 \\ 6 & -1.241391 & -2.448599 & -1.194338 \\ 6 & -0.362018 & -2.823638 & -0.137140 \\ 6 & 0.000000 & 4.299044 & 0.022360 \\ 6 & 2.590216 & 0.976597 & -2.745785 \\ 6 & -2.590216 & -0.976597 & -2.745785 \\ 6 & 0.000000 & -4.299044 & 0.022360 \\ 1 & 0.166318 & 4.609721 & 1.062299 \\ 1 & 0.585985 & 4.941339 & -0.644904 \\ 1 & -1.067952 & 4.442978 & -0.195915 \\ 1 & 3.522899 & 0.497195 & -2.417326 \\ 1 & 2.121896 & 0.306739 & -3.479968 \\ 1 & 2.826654 & 1.931993 & -3.228205 \\ 1 & -3.522899 & -0.497195 & -2.417326 \\ 1 & -2.121896 & -0.306739 & -3.479968 \\ 1 & -2.826654 & -1.931993 & -3.228205 \\ 1 & -0.166318 & -4.609721 & 1.062299 \\ 1 & -0.585985 & -4.941339 & -0.644904 \\ 1 & 1.067952 & -4.442978 & -0.195915 \\ 1 & 1.620110 & 3.258288 & -1.811820 \\ 1 & -1.620110 & -3.258288 & -1.811820 \\ 1 & -1.962616 & -1.046915 & 2.441701 \\ 1 & -2.154691 & 0.720710 & 2.328016 \\ 1 & -2.654212 & -0.311306 & 0.974793 \\ 1 & 2.654212 & 0.311306 & 0.974793 \\ 1 & 1.962616 & 1.046915 & 2.441701 \\ 1 & 2.154691 & -0.720710 & 2.328016 \\ 1 & 0.000000 & 0.000000 & 2.343047\end{array}$


Optimized geometry, AIM data, and coordinates of 8

C2 symmetric

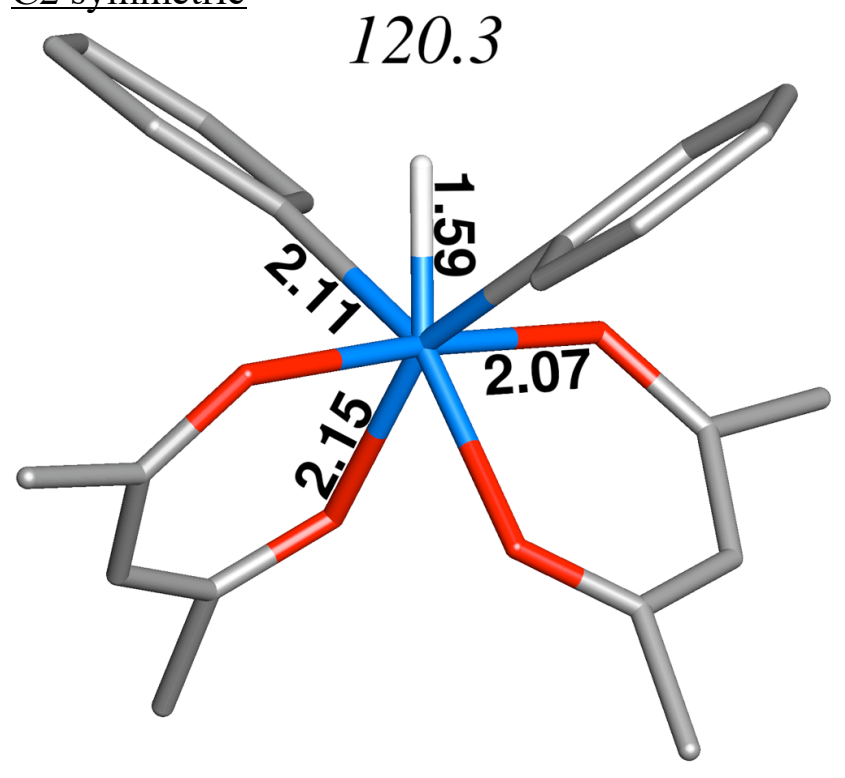

$\mathrm{E}_{S C F}=-1245.42592031$ hartrees

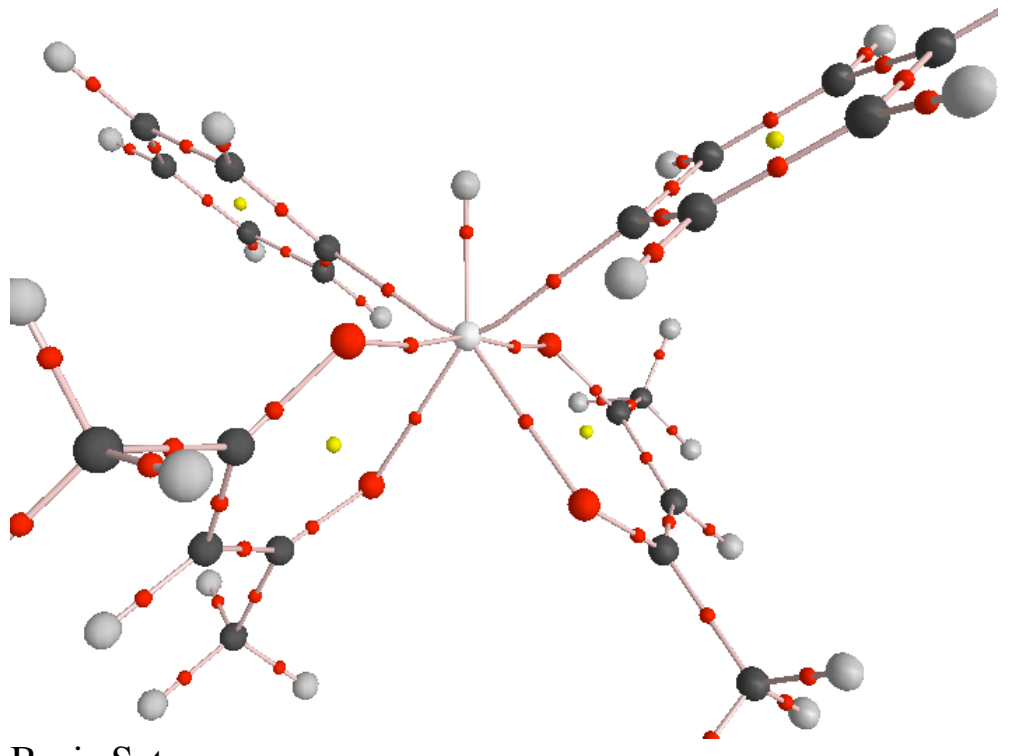

Basis Sets

Opt and Freq

Os: LANL2DZ(f)

O: cc-pvdz

phenyl carbons: cc-pvdz

$\mathrm{H}$ being transferred: cc-pvdz

All other C and H: D95.

AIM analysis

Os: WTBS 
O: cc-pvdz

phenyl carbons: cc-pvdz

$\mathrm{H}$ being transferred: cc-pvdz

All other C and H: D95.

AIM Data
\begin{tabular}{|c|c|c|c|}
\hline & $\rho$ (a.u.) & $\nabla^{2}$ & Distance $(\AA)$ \\
\hline Os-C & 0.121808 & 0.174665 & 1.11952 \\
\hline Os-H $_{\mathrm{t}}$ & 0.158264 & 0.051555 & 1.09078 \\
\hline
\end{tabular}

$\begin{array}{lrrc}76 & 0.000000 & 0.000000 & 0.120197 \\ 8 & 0.000000 & 2.069348 & 0.041501 \\ 8 & 1.355041 & -0.126724 & -1.548966 \\ 8 & -1.355041 & 0.126724 & -1.548966 \\ 8 & 0.000000 & -2.069348 & 0.041501 \\ 6 & 1.827483 & 0.033214 & 1.170131 \\ 6 & -1.827483 & -0.033214 & 1.170131 \\ 6 & 0.644415 & 2.758690 & -0.828321 \\ 6 & 1.507032 & 2.269250 & -1.846748 \\ 6 & 1.808106 & 0.918018 & -2.142675 \\ 6 & -1.808106 & -0.918018 & -2.142675 \\ 6 & -1.507032 & -2.269250 & -1.846748 \\ 6 & -0.644415 & -2.758690 & -0.828321 \\ 6 & 0.434616 & 4.264952 & -0.709015 \\ 6 & 2.779626 & 0.619828 & -3.281217 \\ 6 & -2.779626 & -0.619828 & -3.281217 \\ 6 & -0.434616 & -4.264952 & -0.709015 \\ 6 & 2.743205 & -1.038114 & 1.042247 \\ 6 & 4.011188 & -1.010660 & 1.638068 \\ 6 & 4.418223 & 0.092134 & 2.398704 \\ 6 & 3.529087 & 1.163386 & 2.553600 \\ 6 & 2.263849 & 1.127133 & 1.955176 \\ 6 & -2.743205 & 1.038114 & 1.042247 \\ 6 & -4.011188 & 1.010660 & 1.638068 \\ 6 & -4.418223 & -0.092134 & 2.398704 \\ 6 & -3.529087 & -1.163386 & 2.553600 \\ 6 & -2.263849 & -1.127133 & 1.955176 \\ 1 & 0.000000 & 0.000000 & 1.708323 \\ 1 & 0.760426 & 4.604868 & 0.283388 \\ 1 & 0.985996 & 4.820148 & -1.475351 \\ 1 & -0.635438 & 4.495541 & -0.798521 \\ 1 & 3.669294 & 0.122858 & -2.870918 \\ 1 & 2.310704 & -0.078761 & -3.986753 \\ 1 & 3.089419 & 1.524365 & -3.815957\end{array}$




$\begin{array}{lrrr}1 & -3.669294 & -0.122858 & -2.870918 \\ 1 & -2.310704 & 0.078761 & -3.986753 \\ 1 & -3.089419 & -1.524365 & -3.815957 \\ 1 & -0.760426 & -4.604868 & 0.283388 \\ 1 & -0.985996 & -4.820148 & -1.475351 \\ 1 & 0.635438 & -4.495541 & -0.798521 \\ 1 & 1.984988 & 3.018390 & -2.470486 \\ 1 & -1.984988 & -3.018390 & -2.470486 \\ 1 & 1.587918 & 1.970246 & 2.096407 \\ 1 & 3.821728 & 2.032847 & 3.145192 \\ 1 & 5.403749 & 0.114635 & 2.864443 \\ 1 & 4.686223 & -1.858798 & 1.507372 \\ 1 & 2.449093 & -1.908513 & 0.459539 \\ 1 & -1.587918 & -1.970246 & 2.096407 \\ 1 & -3.821728 & -2.032847 & 3.145192 \\ 1 & -5.403749 & -0.114635 & 2.864443 \\ 1 & -4.686223 & 1.858798 & 1.507372 \\ 1 & -2.449093 & 1.908513 & 0.459539\end{array}$

Optimized geometry, AIM data, and coordinates of 9 C2 symmetric

\section{6}

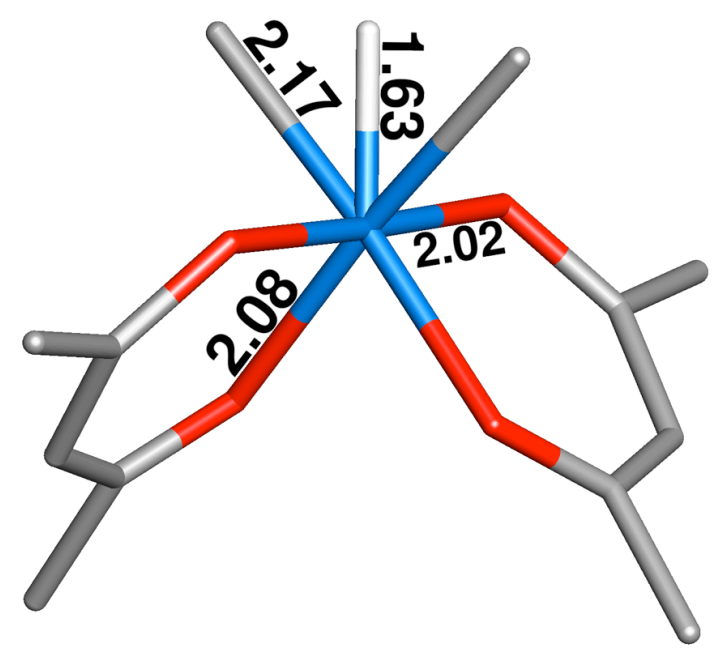

$\mathrm{E}_{S C F}=-899.676826899$ hartrees 


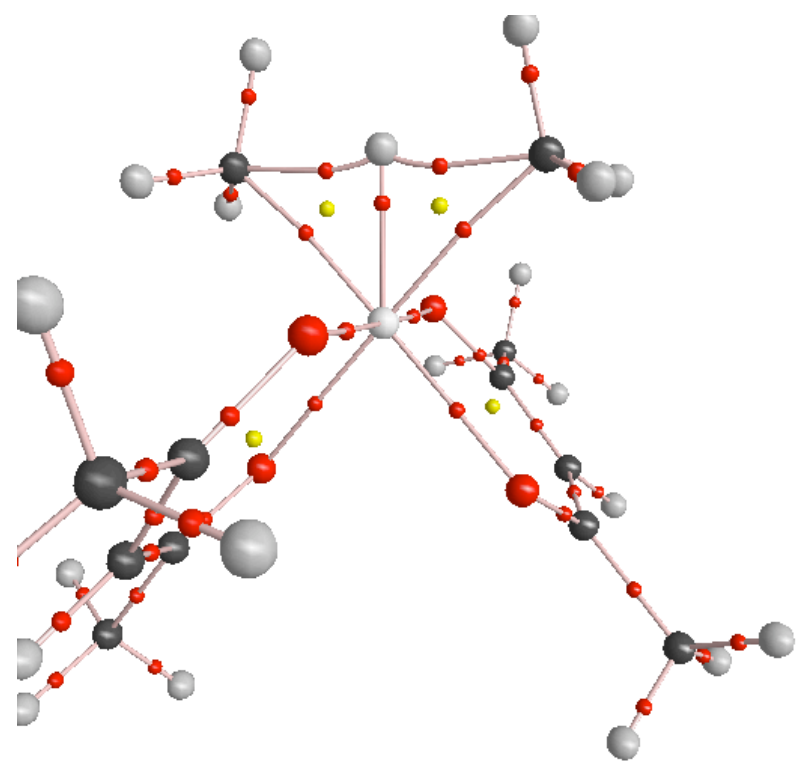

Basis Sets

Opt and Freq

Pt: LANL2DZ(f)

O: cc-pvdz

Ligated carbons: cc-pvdz

$\mathrm{H}$ being transferred: cc-pvdz

All other C and H: D95.

AIM analysis

Pt: WTBS

O: cc-pvdz

Ligated carbons: cc-pvdz

$\mathrm{H}$ being transferred: cc-pvdz

All other C and H: D95.

AIM Data

\begin{tabular}{|c|c|c|c|}
\hline & $\rho$ (a.u.) & $\nabla^{2}$ & Distance $(\AA)$ \\
\hline Pt-C & 0.098688 & 0.157931 & 1.179723 \\
\hline Pt-H $_{\mathrm{t}}$ & 0.130878 & 0.160979 & 1.138435 \\
\hline $\mathrm{C}-\mathrm{H}$ & 0.103731 & -0.030184 & 0.988517 \\
\hline $\mathrm{RCP}$ & 0.095016 & 0.147549 & 1.123083 \\
\hline
\end{tabular}




$\begin{array}{lrrr}78 & 0.000000 & 0.000000 & 0.628507 \\ 8 & -0.221818 & 2.010647 & 0.662715 \\ 8 & 1.396682 & 0.008686 & -0.913767 \\ 8 & -1.396682 & -0.008686 & -0.913767 \\ 8 & 0.221818 & -2.010647 & 0.662715 \\ 6 & 1.555636 & 0.087818 & 2.143435 \\ 6 & -1.555636 & -0.087818 & 2.143435 \\ 6 & 0.384555 & 2.790009 & -0.198561 \\ 6 & 1.303469 & 2.410346 & -1.191539 \\ 6 & 1.762084 & 1.103683 & -1.498243 \\ 6 & -1.762084 & -1.103683 & -1.498243 \\ 6 & -1.303469 & -2.410346 & -1.191539 \\ 6 & -0.384555 & -2.790009 & -0.198561 \\ 6 & 0.000000 & 4.243493 & -0.032605 \\ 6 & 2.778165 & 0.906129 & -2.601155 \\ 6 & -2.778165 & -0.906129 & -2.601155 \\ 6 & 0.000000 & -4.243493 & -0.032605 \\ 1 & 0.127589 & 4.550194 & 1.012199 \\ 1 & 0.593332 & 4.896001 & -0.676358 \\ 1 & -1.061908 & 4.370982 & -0.279790 \\ 1 & 3.668007 & 0.406769 & -2.198212 \\ 1 & 2.361393 & 0.244472 & -3.370884 \\ 1 & 3.074621 & 1.850120 & -3.062816 \\ 1 & -3.668007 & -0.406769 & -2.198212 \\ 1 & -2.361393 & -0.244472 & -3.370884 \\ 1 & -3.074621 & -1.850120 & -3.062816 \\ 1 & -0.127589 & -4.550194 & 1.012199 \\ 1 & -0.593332 & -4.896001 & -0.676358 \\ 1 & 1.061908 & -4.370982 & -0.279790 \\ 1 & 1.709192 & 3.212220 & -1.794730 \\ 1 & -1.709192 & -3.212220 & -1.794730 \\ 1 & -2.014188 & -1.060879 & 1.964998 \\ 1 & -1.335832 & 0.013149 & 3.217031 \\ 1 & -2.167220 & 0.757900 & 1.827864 \\ 1 & 2.167220 & -0.757900 & 1.827864 \\ 1 & 2.014188 & 1.060879 & 1.964998 \\ 1 & 1.335832 & -0.013149 & 3.217031 \\ 1 & 0.000000 & 0.000000 & 2.258724\end{array}$


Optimized geometry, AIM data, and coordinates of $\mathbf{1 0}$

C1 symmetric

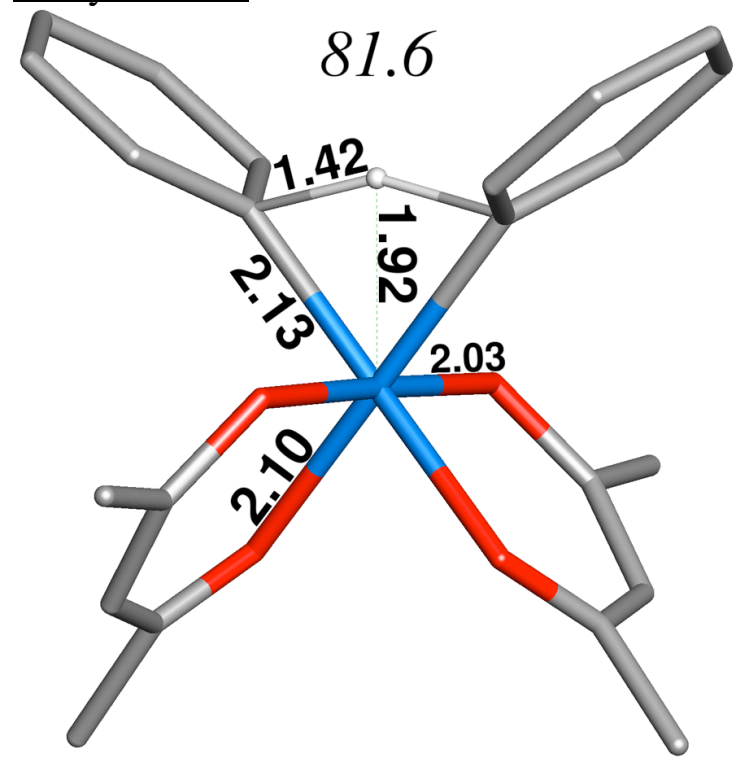

$\mathrm{E}_{S C F}=-1273.19407755$ hartrees

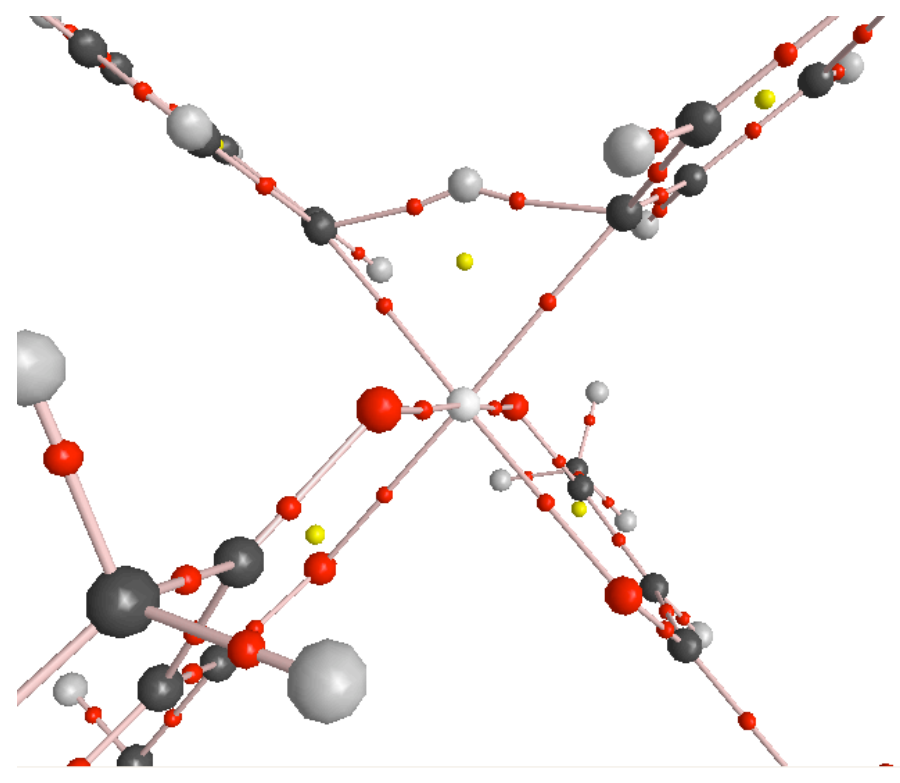


Basis Sets

Opt and Freq

Os: LANL2DZ(f)

O: cc-pvdz

phenyl carbons: cc-pvdz

$\mathrm{H}$ being transferred: cc-pvdz

All other C and H: D95.

AIM analysis

Os: WTBS

O: cc-pvdz

phenyl carbons: cc-pvdz

$\mathrm{H}$ being transferred: cc-pvdz

All other C and H: D95.

AIM Data

\begin{tabular}{|c|c|c|c|}
\hline & $\rho$ (a.u.) & $\nabla^{2}$ & Distance $(\AA)$ \\
\hline Pt-C & 0.110913 & 0.149121 & 1.152977 \\
\hline C-H & 0.122552 & -0.119840 & 0.927599 \\
\hline RCP & 0.073929 & 0.226839 & 1.251005 \\
\hline
\end{tabular}

$\begin{array}{lrrc}78 & -0.000189 & -0.150572 & -0.000057 \\ 8 & 0.940128 & -0.180700 & -1.797364 \\ 8 & 1.188246 & -1.693337 & 0.791264 \\ 8 & -1.191638 & -1.690646 & -0.792062 \\ 8 & -0.940493 & -0.179639 & 1.797257 \\ 6 & 1.223998 & 1.456704 & 0.659546 \\ 6 & -1.221285 & 1.459362 & -0.658836 \\ 6 & 1.818640 & -1.103778 & -2.113033 \\ 6 & 2.359474 & -2.091500 & -1.280468 \\ 6 & 2.059730 & -2.326364 & 0.090031 \\ 6 & -2.064242 & -2.322344 & -0.091032 \\ 6 & -2.363374 & -2.087603 & 1.279630 \\ 6 & -1.820582 & -1.101329 & 2.112630 \\ 6 & 2.257864 & -1.009012 & -3.559367 \\ 6 & 2.819685 & -3.398818 & 0.841994 \\ 6 & -2.826496 & -3.392852 & -0.843443 \\ 6 & -2.259082 & -1.006800 & 3.559196 \\ 6 & 1.217157 & 1.787814 & 2.032633 \\ 6 & 2.205300 & 2.626644 & 2.547411 \\ 6 & 3.200934 & 3.142205 & 1.706571 \\ 6 & 3.207010 & 2.827191 & 0.342432 \\ 6 & 2.226303 & 1.985483 & -0.184473 \\ 6 & -1.213165 & 1.791728 & -2.031605 \\ 6 & -2.199625 & 2.632684 & -2.546144\end{array}$




$\begin{array}{lrrr}6 & -3.194851 & 3.149120 & -1.705363 \\ 6 & -3.202176 & 2.832853 & -0.341522 \\ 6 & -2.223150 & 1.989034 & 0.185142 \\ 1 & 0.001583 & 1.772387 & 0.000699 \\ 1 & 2.619850 & 0.002653 & -3.780352 \\ 1 & 3.045704 & -1.728534 & -3.791783 \\ 1 & 1.399126 & -1.198339 & -4.216140 \\ 1 & 3.515608 & -2.923318 & 1.546198 \\ 1 & 2.123601 & -4.003657 & 1.433491 \\ 1 & 3.390143 & -4.046168 & 0.171883 \\ 1 & -3.523525 & -2.915495 & -1.545313 \\ 1 & -2.132106 & -3.997133 & -1.437463 \\ 1 & -3.396166 & -4.040928 & -0.173356 \\ 1 & -2.615754 & 0.006306 & 3.782095 \\ 1 & -3.050478 & -1.722758 & 3.790543 \\ 1 & -1.401158 & -1.201764 & 4.215417 \\ 1 & 3.098371 & -2.743483 & -1.728818 \\ 1 & -3.103425 & -2.738441 & 1.727748 \\ 1 & 2.217357 & 1.758437 & -1.246290 \\ 1 & 3.973372 & 3.239019 & -0.310617 \\ 1 & 3.968255 & 3.797132 & 2.114430 \\ 1 & 2.202910 & 2.876464 & 3.606227 \\ 1 & 0.448608 & 1.382785 & 2.683436 \\ 1 & -2.215185 & 1.760985 & 1.246747 \\ 1 & -3.968209 & 3.245339 & 0.311498 \\ 1 & -3.960870 & 3.805692 & -2.113025 \\ 1 & -2.196248 & 2.883466 & -3.604730 \\ 1 & -0.444969 & 1.385983 & -2.682382\end{array}$

\section{References:}

Gaussian 03, Revision D.02, Frisch, M. J.; Trucks, G. W.; Schlegel, H. B.; Scuseria, G. E.; Robb, M. A.; Cheeseman, J. R.; Montgomery, Jr., J. A.; Vreven, T.; Kudin, K. N.; Burant, J. C.; Millam, J. M.; Iyengar, S. S.; Tomasi, J.; Barone, V.; Mennucci, B.; Cossi, M.; Scalmani, G.; Rega, N.; Petersson, G. A.; Nakatsuji, H.; Hada, M.; Ehara, M.; Toyota, K.; Fukuda, R.; Hasegawa, J.; Ishida, M.; Nakajima, T.; Honda, Y.; Kitao, O.; Nakai, H.; Klene, M.; Li, X.; Knox, J. E.; Hratchian, H. P.; Cross, J. B.; Bakken, V.; Adamo, C.; Jaramillo, J.; Gomperts, R.; Stratmann, R. E.; Yazyev, O.; Austin, A. J.; Cammi, R.; Pomelli, C.; Ochterski, J. W.; Ayala, P. Y.; Morokuma, K.; Voth, G. A.; Salvador, P.; Dannenberg, J. J.; Zakrzewski, V. G.; Dapprich, S.; Daniels, A. D.; Strain, M. C.; Farkas, O.; Malick, D. K.; Rabuck, A. D.; Raghavachari, K.; Foresman, J. B.; Ortiz, J. V.; Cui, Q.; Baboul, A. G.; Clifford, S.; Cioslowski, J.; Stefanov, B. B.; Liu, G.; Liashenko, A.; Piskorz, P.; Komaromi, I.; Martin, R. L.; Fox, D. J.; Keith, T.; AlLaham, M. A.; Peng, C. Y.; Nanayakkara, A.; Challacombe, M.; Gill, P. M. W.; Johnson, B.; Chen, W.; Wong, M. W.; Gonzalez, C.; and Pople, J. A.; Gaussian, Inc., Wallingford CT, 2004.

LANL2DZ: Hay, P. J.; Wadt, W. R. J. Chem. Phys. 1985, 82, 270 - 283. 
Modified LANL2DZ BS: Couty, M.; Hall, M. B. J. Comp. Chem. 1996, 17, 1359 - 1370. cc-pVDZ: Dunning, T. H. J. Chem. Phys. 1989, 90, $1007-1023$.

D95: Dunning, T. H.; Hay, P. J. Modern Theoretical Chemistry; Schaefer, H. F., III, Ed.; 1976 Plenum: New York, , pp $1-28$.

WTBS: (a) Huzinaga, S.; Miguel, B. Chem. Phys. Lett. 1990, 175, 289 -291. (b) Huzinaga, S.; Klobukowski, M. Chem. Phys. Lett. 1993, 212, 260 - 264.

AIM images for altering geometric parameters of 9
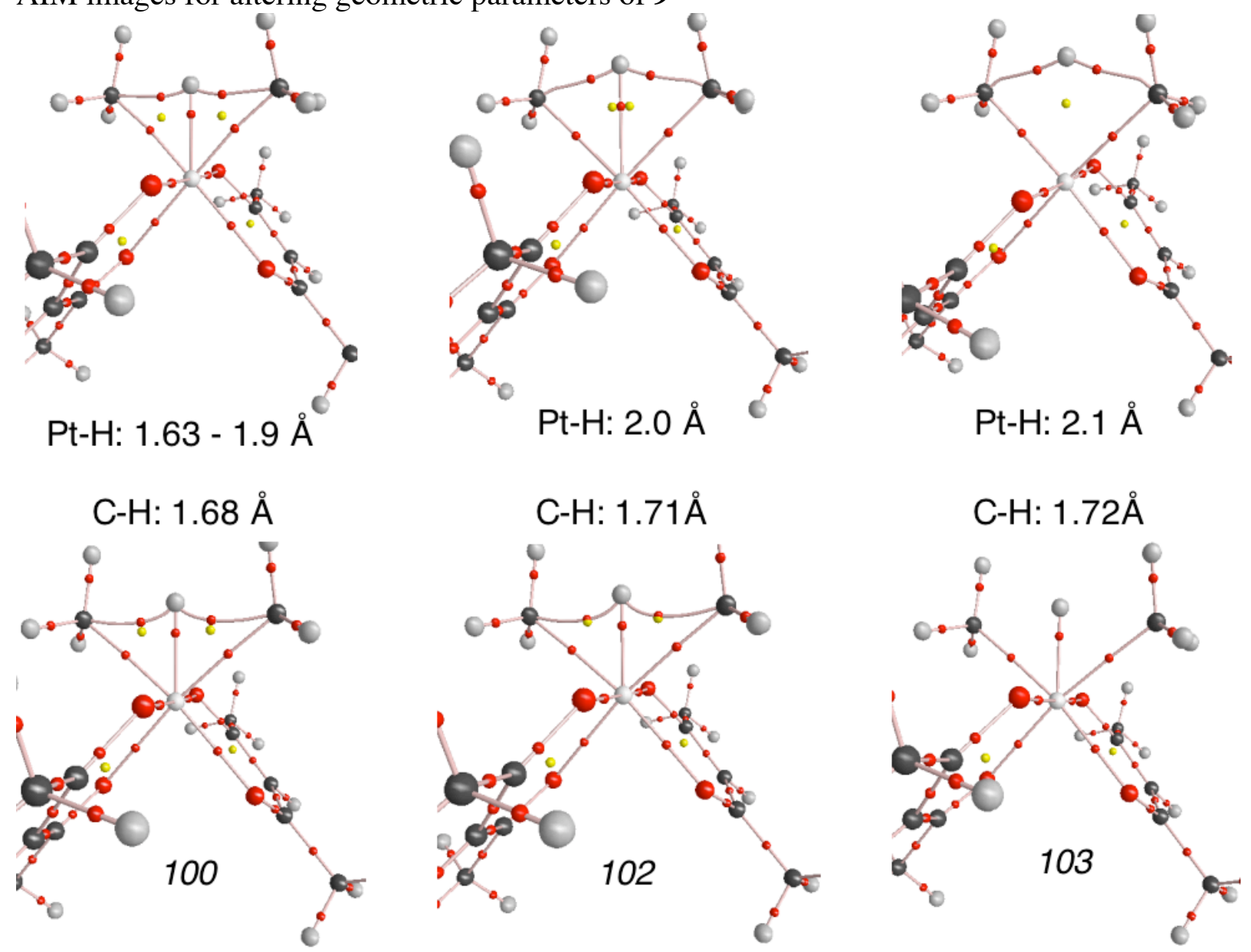

In the AIM analysis critical points are defined in the gradient field as $\nabla \rho(\mathbf{r})=0$. These critical points are differentiated through analysis of the Hessian matrix for a given CP; upon diagonalization, the "signature" (number of curvatures, sum of the character of the diagonal elements) of the $\mathrm{CP}$ can be assigned. For a bond $\mathrm{CP}(\mathrm{BCP})$, there are three curvatures with two negative and one positive eigenvalues $(+1-1-1=-1)$; thus the signature of a BCP is $(3,-1)$. A $\mathrm{BCP}$ is essential for a direct interaction between atoms. The signature of a ring critical point $(\mathrm{RCP})$ is $(3,+1)$ and of a cage critical point $(\mathrm{CCP}),(3,+3)$. 